\title{
Faire écrire des élèves dans une visée philosophique : scénario pédagogique et regard du psychologue
}

\section{Training students to write with a philosophical aim: pedagogical scenario and psychological perspective}

\author{
Hélène Maire ${ }^{1,2}$, Emmanuèle Auriac-Slusarczyk ${ }^{2} \&$ Julie Pironom $^{2}$ \\ 1 Laboratoire Lorrain de Psychologie et Neurosciences (2LPN, EA 7489), Université \\ de Lorraine, Nancy, France. \\ 2 Laboratoire Activité, Connaissance, Transmission, Education (ACTé, EA 4281), \\ Université Clermont Auvergne, Clermont-Ferrand, France.
}

Contact : helene.maire@univ-lorraine.fr

Pour citer cet article :

Maire, H., Auriac-Slusarczyk, E., \& Pironom, J. (2019). Faire écrire des élèves dans une visée philosophique : scénario pédagogique et regard du psychologue. Studia Universitatis Babes-Bolyai. Philosophia. doi:10.24193/subbphil.2019.3.05

Résumé : Avec l'essaimage international de la P4C (Philosophy FOR Children), les bénéfices de cette pratique orale et collective pour le développement des enfants ont été démontrés, et ce dans des domaines variés et transversaux. Ces effets bénéfiques sont-ils transférables et objectivables dans des écrits individuels d'élèves de 13 à 14 ans ? Nous esquisserons des pistes pour répondre à cette question en présentant un dispositif pédagogique original de $\mathrm{P} 4 \mathrm{C}$, Philo \& Carto, qui s'appuie sur des œuvres d'art comme supports visant à amorcer le dialogue en communauté de recherche. Les résultats d'une première expérimentation menée avec ce dispositif seront présentés et discutés, et des pistes pédagogiques seront proposées.

Mots-clés : philosophie pour enfants ; psychologie de l'éducation ; écriture ; transfert d'apprentissage

\begin{abstract}
With the international swarming of the oral and collective P4C (Philosophy FOR Children) practice, its benefits for children's development have been demonstrated in various and transversal fields. Are these beneficial effects transferable and quantifiable in individual writings of students aged 13 to 14 years? We will outline some directions to answer this question by presenting an original educational P4C tool, Philo \& Carto, which is based on artworks as anchors to initiate the dialogical community of inquiry. The results from a first experiment will be presented and discussed, and pedagogical directions will be proposed.
\end{abstract}

Keywords: philosophy for children; educational psychology; writing; transfer of learning 


\section{Cadre théorique}

\section{1. a. L'essaimage des dialogues philosophiques et leurs apports pour le développement des enfants}

Les dialogues philosophiques (P4C pour «Philosophy FOR Children », DVP pour « Discussions à Visée Philosophique », ou encore PPE pour « Philosophie Pour Enfants ») est une praxis ${ }^{1}$ pédagogique plus ou moins cadrée qui a émergé aux Etats-Unis dans les années 1970/1980². Matthew Lipman, enseignant universitaire en philosophie, avait alors constaté que les exercices d'inférence logique ou de validité des raisonnements arrivaient trop tard (ou n'arrivaient jamais !) dans le cursus de ses étudiants, impactant dès lors assez peu leur manière de se poser les problèmes ; en revanche, les jeunes enfants étaient, selon lui, en capacité de tirer profit d'un enseignement de l'art de raisonner à condition que celui-ci leur soit dispensé assez tôt dans leur développement. Le constat était fait par ailleurs que tout enfant est animé par le double besoin de vivre pleinement le pouvoir de la pensée et de questionner la valeur des conceptions de l'existence qui l'entourent sur un mode autre que scolaire ${ }^{3}$. De là, la pratique de la philosophie pour enfants a émergé, puis s'est consolidée, autour de deux spécificités majeures : 1) présupposer chaque élève comme un «interlocuteur valable », en capacité de se poser des questions importantes, et ce dès l'âge préscolaire de 5 ans environ, et 2) assurer, par le biais du seul canal oral, qu'un cheminement réflexif collectif soutient et étaye les pensées individuelles.

Dans cette perspective, le protocole de dialogue philosophique pour enfants proposé par Lipman s'articule en trois étapes. Durant un premier temps de lecture partagée, chaque élève lit à voix haute, à tour de rôle, un extrait d'un chapitre de roman. Ensuite, au cours de la cueillette des questions, les élèves s'attachent à essayer de comprendre le/les sens du chapitre lu jusqu'à interroger les mots ou les situations décrites dans le roman, ainsi que les acquis, croyances, traditions et préjugés qui y sont charriés ; chacun formule ainsi son interrogation sur le monde. Enfin, une question retravaillée pour être partagée (collective) est extraite à partir de l'ensemble des questions (individuelles) formulées, et le dialogue philosophique en communauté de recherche s'instaure et se déploie, dans une construction à la fois personnelle et sociale du sens. Différentes variations autour de ce protocole princeps, s'en inspirant ou s'en éloignant à des degrés variables, ont été proposées, notamment, en France, par Jacques Lévine

\footnotetext{
${ }^{1}$ Le terme « praxis » est utilisé pour souligner le fait que cette pratique établit une relation entre la réflexion et l'action, et que son apprentissage y est vu comme un processus en cours plutôt que comme un produit.

${ }^{2}$ Lipman, M., Sharp, A. M., \& Oscanyan, F. S. (1980).

${ }^{3}$ Lévine, J., Chambart, G., \& Sillam, M. (2005).
} 
et Michel Tozzi ${ }^{4}$, mais aussi François Galichet ${ }^{5}$, Marielle Rispail ${ }^{6}$ et Emmanuèle AuriacSlusarczyk ${ }^{7}$.

L'essaimage progressif de cette pratique, que ce soit sous la forme du protocole lipmanien «pur» ou de ses alternatives, est allé de pair avec la formulation d'objectifs ambitieux, et diversifiés, puisque ces derniers visent à la fois le développement de la compréhension éthique (exercer «des esprits libres et réflexifs, capables de résister à différentes formes de propagande, de fanatisme, d'exclusion et d'intolérance $»^{8}$ ), de l'esprit critique, des capacités argumentatives, logiques, ou encore de la créativité. Une véritable inflation des missions lui étant assignées s'est opérée, conduisant à la nécessité de passer au crible de la science les vertus conférées à cette pratique. Si l'évaluation des facultés supposément travaillées par la DVP demeure délicate, un nombre croissant de travaux en psychologie et en sciences de l'éducation, a mis peu à peu en lumière l'intérêt de cette pédagogie dans des domaines variés, parmi lesquels : une favorisation des comportements prosociaux $^{9}$, de l'estime de soi scolaire ${ }^{10}$, des compétences sociales ${ }^{11}$ et d'autorégulation ${ }^{12}$, mais aussi des gains chiffrables en points de quotient intellectuel ${ }^{13}$ ou de maturité intellectuelle globale, ainsi que de meilleures capacités argumentatives et de raisonnement ${ }^{14}$.

Ainsi, les facultés facilitées par la praxis philosophique sont diverses et transversales. Certaines de ces facultés sont à l'œuvre simplement par l'invitation à réfléchir que représente le dispositif (autrement dit, sa visée philosophique) : le questionnement naïf découlant d'heureux décalages et le «simple» art de l'étonnement, la production de concepts et de distinctions de plus en plus fines entre eux, la problématisation de sa pensée et la construction d'hypothèses, notamment. D'autres facultés sont plutôt développées par la modalité particulière du dispositif, qui passe par l'expression orale en groupe et la construction collective du sens portée par la discussion : celle-ci prépare au dialogue de haut niveau (Daniel, 2003) ${ }^{15}$ tant elle active puis présuppose, au fil du développement en âge, l'activation de modalités

\footnotetext{
4 Tozzi, M. (2005).

${ }^{5}$ Galichet, F. (2007).

${ }^{6}$ Rispail, M., Calistri, C., Martel, C., \& Bomel-Rainelli, B. (2007).

${ }^{7}$ Auriac-Slusarczyk, E., \& Colletta J.-M. (2013).

${ }^{8}$ Cet objectif a été formulé par l'UNESCO en 2007.

${ }^{9}$ Colom, R., Moriyón, F. G., Magro, C., \& Morilla, E. (2014).

10 Trickey, S., \& Topping, K. J. (2006).

${ }^{11}$ Giménez-Dasí, M., Quintanilla, L., \& Daniel, M.-F. (2013).

${ }^{12}$ Heron, G., \& Cassidy, C. (2018).

${ }^{13}$ García-Moriyón, F., Rebollo, I., \& Colom, R. (2005).

${ }^{14}$ Pour des revues plus exhaustives des effets positifs de la philosophie pour enfants, nous renvoyons à trois synthèses : Auriac-Slusarczyk, E., \& Maire, H. (à paraître) ; Gregory, M.-R., Haynes, J., \& Murris, K. (2016) ; Millett, S., \& Tapper, A. (2012).

15 Daniel, M.-F. (2003).
} 
argumentatives variées, favorisant, entre autres, l'évocation d'images mentales, l'usage d'analogies ou de métaphores, la mobilisation d'exemples se détachant de la simple anecdote personnelle ${ }^{15}$ comme de l'habitus du récit de soi. Autrement dit, sont à l'œuvre des compétences linguistiques et logiques, largement intriquées ou articulées les unes aux autres, mais aussi “ $a$ dimension of childhood power and creativity that is completely missed by the people who think that children begin with intellectual weakness and then gradually mount up to higher and higher echelons of strength and understanding" 16 , c'est-à-dire des compétences réflexives et créatives.

Par ailleurs, l'ensemble de ces facultés recouvrent, au moins partiellement, les compétences travaillées et évaluées à l'écrit par certaines disciplines scolaires, en français puis en philosophie notamment. Dès lors, on peut s'interroger : un transfert de ces facultés s'opèret-il lorsqu'on propose aux élèves, non plus de discuter collectivement, mais d'écrire individuellement à propos d'une question philosophique ? Le statut d' « interlocuteur valable » ou le Moi, instance symbolique civilisatrice ${ }^{17}$ instauré par la discussion philosophique, est-il susceptible, lorsqu'il est exercé à l'oral, de contribuer à déverrouiller certains blocages liés à l'écrit, chez des élèves en passe de devenir des « scribes valables »? En bref, un transfert des facultés exercées collectivement à l'oral s'opère-t-il quand il s'agit d'écrire, seul(e) face à sa feuille blanche?

\section{1. b. Caractériser a minima l'écriture philosophique d'élèves}

Etudier le transfert, de l'oral à l'écrit, de facultés à philosopher implique de circonscrire le périmètre de ce qu'on peut (ou non) qualifier de «philosophique ». Philosopher implique $a$ minima de questionner ce qui d'ordinaire ne l'est pas, ou pas de la même manière : «à partir du moment où je me demande : mais est-ce que vraiment j'ai raison de penser que (...) ? à partir du moment où je me pose la question : 'Est-ce que c'est vraiment ça ?' Là, je fais de la philosophie proprement dite » A quelle(s) condition(s) un écrit peut-il être désigné comme étant «philosophique »? Examinons dans un premier temps les critères permettant de dire d'une discussion qu'elle est philosophique. Il faut pour cela :

(...) qu'elle ; étudie un concept ou un thème philosophique ; s'appuie sur des éléments logiques (inférences, syllogismes, comparaisons, relations cause-effet, partie-tout, etc.); conduit à d'autres questionnements au lieu de fournir des réponses ; présuppose chez les participantes et participants une pensée autonome (construction originale), une ouverture d'esprit eu égard aux diverses perspectives, un sens critique (recherche d'objectivité, sensibilité au contexte, autocorrection) et une pensée créatrice (élaboration de solutions pertinentes, d'alternatives nouvelles, etc.); suppose

\footnotetext{
${ }^{16}$ Lipman, M. (1991).

${ }^{17}$ Lévine, J. (2007).
} 
des attitudes coopératives (écoute, respect, tolérance, compromis, acceptation d'être influencé par l'autre, etc.). ${ }^{18}$

Si l'ouverture d'esprit et les attitudes coopératives semblent spécifiques au genre oral, nombre de ces critères sont communs aux genres écrit et oral. Prenant appui sur ces premiers éléments, définir le genre de l'écrit philosophique reste un exercice délicat, d'autant qu'il n'est pas enseigné dans le système scolaire français avant l'âge de 17 ans. A minima, on peut toutefois en délimiter certains contours. Ne pouvant être réduit au seul genre argumentatif, l'écrit philosophique se situerait à la croisée de la dissertation (aspects linguistiques et logiques), de l'invention (par aspects créatifs) et de l'«introspection située » (aspects réflexifs). Pour être philosophique, un texte présenterait l'état d'une question prédéfinie sous différents angles, en les examinant, les comparant, les distinguant, les explorant via un cheminement intellectuel plus ou moins aventureux. Une part impressionniste et singulière (c'est-à-dire ce que l'on pense, ressent, entraperçoit) accompagne l'activation des connaissances partagées par le commun. Par le truchement de postulats (savoirs partagés, doxa), d'hypothèses originales et/ou partagées, et d'exemples, certaines idées seraient davantage explorées pour atteindre parfois le rang de concepts (par exemple, «les émotions», «le temps »). Ce cheminement intellectuel ${ }^{19}$ serait alimenté par certains raisonnements analogiques susceptibles de favoriser des décalages de pensée féconds, mais aussi de se substituer, dans certains cas, aux mots eux-mêmes si ceux-ci ne «viennent» pas à l'élève in situ. Ce cheminement convoquerait aussi des anecdotes personnelles, nécessaires pour retraduire le caractère expérientiel de toute activité de pensée ${ }^{20}$, et pouvant tout à la fois venir appuyer (exemples) ou au contraire réfuter (contre-exemples) l'un des arguments émis. Dans une perspective minimaliste de définition, on s'accorde, avec les philosophes et les professeurs de philosophie, pour dire qu'un texte est philosophique s'il :

(...) développe des propositions, selon un certain ordre de succession de phrases avec un certain style (compact ou dilué, elliptique ou bavard) et dans une certaine atmosphère (un ton polémique ou une certaine sérénité), un ensemble d'idées, d'hypothèses, d'avis, de jugements qui peuvent tous être plus ou moins bien reliés, illustrés, exposés et argumentés (p. 347, c'est nous qui marquons en gras certains mots) $)^{21}$.

Aussi, par la suite, nous appuierons nous sur ces balises définitionnelles produites par les philosophes, ce dans une visée formative, pour caractériser les textes produits par les élèves.

\section{Objectifs et méthodologie}

\footnotetext{
${ }^{18}$ Daniel, M.-F. (2003, p.241).

${ }^{19}$ Le terme d'« aventure intellectuelle » a été proposé pour décrire ce cheminement par Beausoleil, J. et Daniel, M.-F. (1991).

${ }^{20}$ Dewey, J. (1958) ; Dewey, J. (1963).

${ }^{21}$ Choulet, P., Folscheid, D., \& Wunenbuger, J.-J. (1992).
} 
Deux premières études explorant les conditions d'âge ${ }^{22}$ et les effets du thème, du support et des consignes choisis pour amorcer l'écriture ${ }^{23}$ ont été menées en amont pour vérifier si des élèves étaient aptes à produire des écrits philosophiques, en suivant une approche de psychologie expérimentale du développement. Le travail présenté s'inscrit dans la continuité de ces recherches antérieures, et vise à comprendre dans quelle mesure une pratique régulière de la DVP peut (ou non) favoriser cette aptitude à écrire chez des élèves de 13-14 ans.

Nous présentons à la fois le dispositif pédagogique singulier utilisé, nommé Philo \& Carto, assorti de la méthodologie que nous avons déployée pour saisir d'éventuelles différences de performances en termes de qualité rédactionnelle et philosophique, chez des élèves ayant bénéficié de ce dispositif, en comparaison à des élèves n’en ayant pas bénéficié.

Trois groupes de collégiennes et collégiens ont été suivis et étudiés. Ils étaient en tous points comparables : milieu de niveau socio-économique similaire plutôt médian, secteur intermédiaire entre urbain et rural, ratios identiques de filles et de garçons dans les classes, moyennes en français équivalentes $(12,8,13,5$ et 13,7 respectivement pour les trois groupes qui vont être présentés ci-après). Les trois groupes ont été soumis à une expérience pédagogique différente.

- Le premier groupe (dit «philosophant», 4 classes) a pratiqué la DVP selon le dispositif Philo \& Carto en classe de $4^{\text {ème }}$ et avait pratiqué la DVP selon un dispositif lipmanien classique au cours des deux années précédentes, en classes de $6^{\text {ème }}\left(11-12\right.$ ans) et de $5^{\text {ème }}$ (12-13 ans).

- Le deuxième groupe (dit «semi-philosophant», 4 classes) avait seulement pratiqué la DVP selon un dispositif lipmanien classique au cours des deux années précédentes.

- Enfin, le troisième groupe (dit « contrôle » ou «non philosophant », 4 classes) n'avait jamais pratiqué la DVP.

Ce type de méthodologie fournit des conditions de recueil de données optimales, puisqu'équilibrées au regard d'une double contrainte : elles sont à la fois écologiques (c'est-àdire ayant lieu dans des conditions réelles de classe) et contrôlées (les groupes ne variant que par le facteur d'influence manipulé, à savoir le dispositif pédagogique introduit, d'éventuelles différences ultérieures de performances entre eux ne pourront être attribuées qu'à ce facteur).

\footnotetext{
${ }^{22}$ Auriac-Slusarczyk, E., Thebault, C., Slusarczyk, B., Daniel, M. F., \& Pironom, J. (2018).

${ }^{23}$ Auriac-Slusarczyk, E., Maire, H., Thebault, C., \& Slusarczyk, B. (2019).
} 


\section{2.a. La pratique philosophique des classes philosophant à travers le dispositif Philo \&} Carto

Dans les classes «philosophant», le dispositif oral utilisé, Philo \& Carto $^{24}$, s'inspirait du protocole classique lipmanien, mais en l'aménageant de façon à inciter les élèves à associer les idées philosophiques (concepts) à des images (symboles). Ces associations aboutissaient in fine à des sortes de cartographies à la fois conceptuelles et artistiques. Pour chaque discussion, une œuvre d'art était utilisée comme support d'ancrage à la discussion. Les œuvres retenues étaient les suivantes : Le Géographe de Johannes Vermeer, La lectrice soumise de René Magritte, Vive l'amour de Niki de Saint-Phalle, Paysage anthropomorphe de Joos de Momper, La Tour de Babel de Pieter Brueghel, Passage de Robert et Shana Parkeharisson, Pandora de John William Waterhouse, et Storm King Wall d'Andy Goldsworthy. Chaque discussion était animée par des enseignantes de diverses disciplines (français, histoire, documentation), travaillant en binôme et épaulées par une professeure d'arts plastiques. Toutes étaient volontaires et avaient été formées au dispositif directement par la formatrice à l'origine de cette variante alliant discussion philosophique et pratique artistique, Cathy Thebault ${ }^{25}$ qui a rodé le dispositif dans des classes d'école élémentaire.

Pour chaque DVP tenue dans toutes les classes de $4^{\text {ème }}$ du groupe «philosophant », le déroulé comportait systématiquement cinq étapes. A partir de l'observation de l'une de ces œuvres, la cueillette de questions philosophiques s'amorçait. L'une d'entre elles était choisie ${ }^{26}$, puis la discussion autour de cette question se mettait en place en communauté de recherche. Au fil de cette discussion, des idées philosophiques ou «concepts » émergeaient, et une dizaine d'entre eux étaient listés puis retenus au fil de l'échange collectif, le groupe d'élèves, aidé des deux enseignantes animatrices, déterminant les concepts les plus représentatifs des échanges tenus en se limitant à dix concepts. Par exemple, à propos de l'œuvre Vive l'amour de Niki de Saint-Phalle, les concepts d'incertitude, de sentiments et de rupture ont été extraits. Les enseignantes utilisaient les propos des élèves et aidaient ces derniers à trouver le mot-clef, de synthèse, quand ces derniers n'y accédaient pas directement. Un retour réflexif portant un

\footnotetext{
${ }^{24}$ Thebault, C. (à paraître).

25 Thebault, C. (2015).

${ }^{26}$ Le choix de la question retenue est une étape du protocole lipmanien qui peut paraître délicate, voire périlleuse. Certains critères définissant ce qu'est une question porteuse de sens philosophique peuvent guider judicieusement ce choix. Une question est dite philosophique «(...) lorsqu'elle: «concerne le "pourquoi » plutôt que le « comment »; questionne les concepts (par exemple : " qu'est-ce que... ? » et « que veut dire... ? ») ; s'élabore autour de l'origine, de la cause, des conséquences, des relations (logiques et linguistiques) entre les mots, les concepts, les idées (par exemple : « d'où vient... ? » et « que va-t-il arriver si... ? »); remet en question les acquis, les traditions, les préjugés (par exemple : "est-il vrai que ?» et «pourquoi pense-t-on que ... ? ») ; cherche des justifications aux énoncés des pairs (par exemple : "pourquoi dis-tu cela? ») »(Daniel, M.-F., 2005, p.34).
} 
nouveau regard sur l'œuvre était ensuite proposé aux élèves, cette fois-ci dans le but de sélectionner une dizaine de fragments picturaux de l'œuvre. Pour exemple, concernant l'œuvre de La lectrice soumise de René Magritte, la main, l'œil ou le livre pouvaient être ciblés, mais aussi la bouche, la coiffure comme le fond du tableau, éventuellement séparés en ciel et sol avec un trait horizontal rajouté à l'œuvre support pour évoquer l'opposition du ciel et de la terre. Enfin, au cours d'une dernière étape, les élèves étaient invités à mettre en correspondance les concepts, abstraits, issus de la première phase de discussion, aux éléments sensibles, perçus, issus du deuxième regard porté sur l'image de l'œuvre. Cette mise en correspondance pouvait aboutir à des sortes de «métaphores situées », plus ou moins évocatrices. Ainsi, dans notre exemple portant sur l'œuvre Vive l'Amour de Nikki de Saint Phalle, l'incertitude associée au nuage était verbalisée sous l'expression métaphorique « le nuage de l'incertitude »; de même, les élèves avaient créé les expressions « le sol des sentiments » ou « la pomme de la rupture ». Cette dernière phase aboutissait à la réalisation d'une œuvre plastique collective, doublement inspirée d'une analyse interprétative de l'œuvre source travaillée via la discussion philosophique tenue. La Figure 1 reproduit la cartographie élaborée par les élèves d'une des classes à partir de l'œuvre Vive l'Amour de Nikki de Saint Phalle ${ }^{27}$. La Figure 2 reproduit la cartographie composée après discussion collective inspirée de l'œuvre La lectrice soumise de René Magritte.

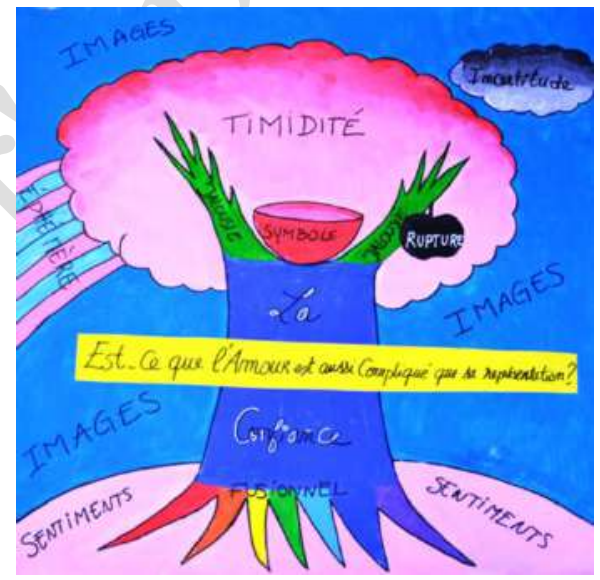

Figure 1 : Création graphique composée après discussion collective à partir de l'œuvre Vive l'Amour et autour de la question collective Est-ce que l'amour est aussi compliqué que sa représentation?

A partir de la question (Est-ce que l'amour est aussi compliqué que sa représentation ?) retenue suite à la cueillette, la communauté de recherche a discuté et extrait certains concepts : sentiments, timidité, confiance, incertitude, éphémère. Puis, lors de la phase de retour à l'image, furent sélectionnés certains fragments picturaux présents dans l'œuvre initiale de Saint-Phalle : tronc, feuille, ciel. La mise en correspondance de chaque idée/concept avec un fragment

\footnotetext{
${ }^{27}$ Le lecteur/la lectrice trouvera facilement le visuel sur Internet.
} 
pictural a donné lieu à une production plastique collective finale, légendée des expressions ou des mots évoquant le travail philosophique accompli ${ }^{28}$.

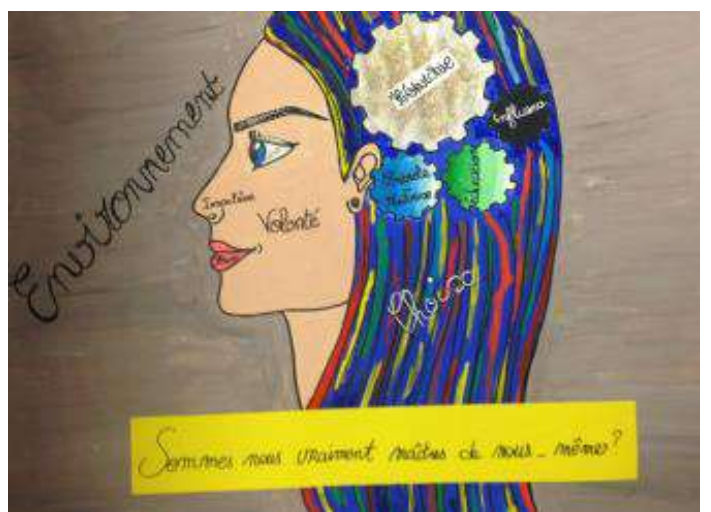

Figure 2 : Création graphique élaborée après discussion collective à partir de l'œuvre La lectrice soumise et autour de la question collective Sommes-nous maîtres de nous-mêmes?.

Inspirée de La lectrice soumise de René Magritte, la question collective (Sommes-nous vraiment maîtres de nous-mêmes $?^{29}$ ) retenue suite à la cueillette, la communauté de recherche a discuté et fléché certains concepts : choix, plaisir, impulsion, environnement. Puis, lors de la phase de retour à l'image, les élèves ont sélectionné certains fragments picturaux, extraits ou inspirés de l'œuvre de Magritte: mèches de cheveux, lèvre, nez, fond. La mise en correspondance de chaque idée : concept (la mèche du choix, la lèvre du plaisir, le nez de l'impulsion), avec un fragment d'image a donné lieu à la production de la cartographie collective, œuvre plastique finale.

Les élèves n'ayant pas bénéficié du dispositif Philo \& Carto (c'est-à-dire nos groupes « contrôle » et « semi-philosophant ») n'ont pas travaillé sur ces œuvres d'art et ont, au cours de leur scolarité au collège, été confrontés à des traitements différents concernant la DVP :

- les classes contrôle, n'ont jamais pratiqué la philosophie.

- les classes semi-philosophant, avaient philosophé en classe de $6^{\text {ème }}$ puis de $5^{\text {ème }}$, et ne furent soumis à aucun travail de philosophie l'année du test (4 $\left.{ }^{\text {ème}}\right)$.

\section{2. b. L'évaluation de la qualité rédactionnelle et philosophique des textes}

Afin d'apprécier d'éventuelles différences dans les compétences écrites des élèves sous l'effet du dispositif, les élèves de l'ensemble des groupes étaient invités à produire un écrit philosophique libre en milieu d'année scolaire. Cet écrit portait sur le thème du rêve et se faisait

\footnotetext{
${ }^{28}$ Slusarczyk, B. et collaborateurs (2017).

${ }^{29}$ Il est à noter la stupéfiante diversité des questions soulevées par les élèves à partir d'une seule et même œuvre d'art. Par exemple, l’œuvre La lectrice soumise de René Magritte a suscité des questions aussi variées que : Peuton être soumis par la culture? Pourquoi les femmes sont-elles toujours représentées en situation de soumission? Pourquoi lire fait peur? En quoi la lecture peut-elle nous soumettre? L'écriture reflète-t-elle la réalité ? Peut-on être soumis sans le savoir? Doit-on croire tout ce qu'on lit?
} 
à partir d'une œuvre d'art, ancrage référentiel servant à amorcer l'écriture : Le Chasseur d'étoiles, de Richard Texier. A la manière de Célestin Freinet et ses «textes libres » ${ }^{30}$, la tâche était ainsi cadrée mais la consigne, ouverte, laissait à la pensée des élèves une confortable latitude pour tâtonner et/ou se déployer. Ces textes avaient vocation à être édités dans une revue à destination d'adolescents ${ }^{31}$, donc lus par des pairs ; les écrits étaient ainsi motivés, et dotés d'une fonction de communication directe puisqu'ils s'adressaient à quelqu'un (d'autre que l'enseignant). Les instructions orales que les enseignants et enseignantes fournissaient à leurs élèves étaient les suivantes : "Votre classe a été sélectionnée pour écrire un texte pour la revue Adolescence et Société. Vous avez donné votre accord. Il s'agit d'écrire le mieux possible. Vous ne lirez pas les textes. Ce sont les éditeurs de la revue qui liront les textes. Ils sont très intéressés. ». Il s'agissait également d'expliquer aux élèves qu'il s'agissait d'un écrit individuel et qu'ils ne pouvaient recevoir aucune aide : «L'éditeur l'interdit. ». L'impératif de propreté était aussi mentionné pour faciliter la lecture ultérieure : «Il vaut mieux éviter les ratures. Il faut écrire lisiblement ». La consigne écrite était lue en collectif avec les élèves, afin de lever leurs éventuelles incompréhensions. Le feuillet fourni incluait une place pour l'avant-texte ${ }^{32}$, destiné à pouvoir coucher quelques idées préalables au bouillon, et trois pages pour le texte (voir Figure 3). Pour l'avant-texte, les enseignants et enseignantes précisaient à l'oral aux élèves : «C'est une sorte de page brouillon. On peut y raturer, y noter des éléments avant d'écrire ». Les élèves renseignaient eux-mêmes leurs nom, prénom et âge avant la tâche d'écriture, et leur date de naissance immédiatement après. Le temps d'écriture, pris sur le temps scolaire, n'était pas contraint; il ne dépassait toutefois pas les 50 minutes, distribution des feuillets et passation de consignes comprises. Une fois récoltées, toutes les copies recueillies ont été numérisées et déposées sur la plateforme en libre accès: https://philosophemes.msh.uca.fr.

\footnotetext{
${ }^{30}$ Schneuwly, B. (1994).

${ }^{31}$ Cette revue est consultable en ligne : https://madmagz.com/fr/magazine/1324694\#/

${ }^{32}$ Expression utilisée par les généticiens du texte, pour insister sur la valeur textuelle, organisationnelle, et conceptuelle du brouillon (Fenoglio, I., \& Boucheron-Pétillon, S., 2002).
} 

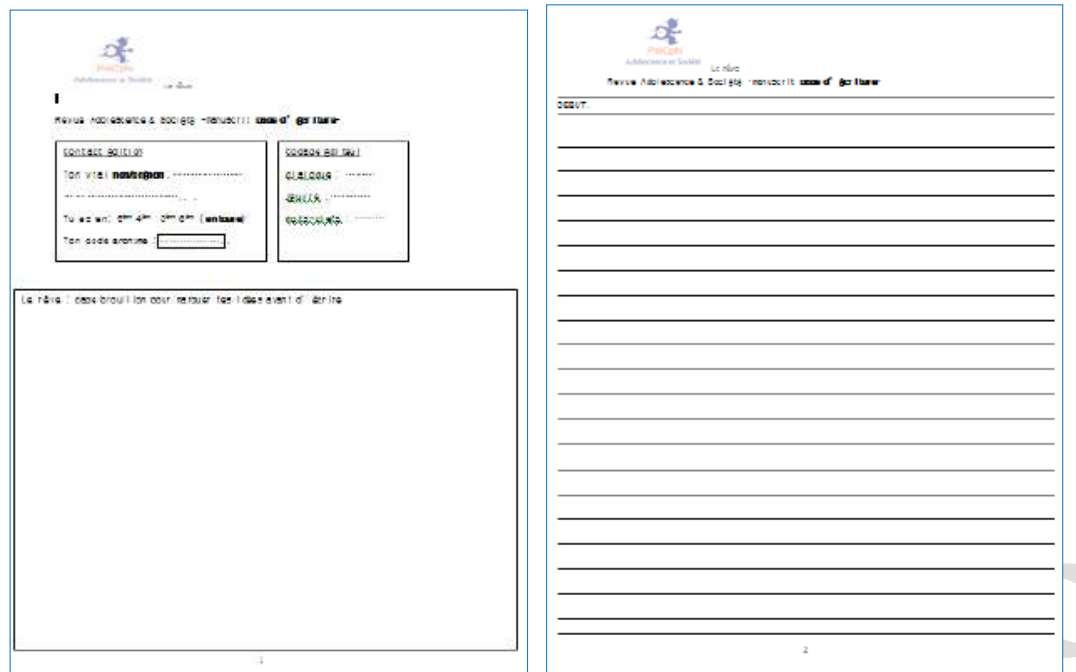

Figure 3 : Exemple de feuillets d'écriture fournis aux élèves : informations relatives à l'élève, avant-texte, première page de texte.

151 copies de collégiennes et collégiens, âgés de 13 à 14 ans, ont ainsi été évaluées :

- 50 issues d'élèves des classes «philosophant » expérimentant la philosophie depuis plus de 2 ans ;

- 72 des classes « semi-philosophant » c'est-à-dire l'ayant expérimenté les deux années précédentes mais ne l'expérimentant plus l'année du test d'écriture (classes semi-philosophant)

- 29 des classes contrôle.

L'avant-texte (c'est-à-dire la mise en forme des idées) ${ }^{33}$ comme le texte, et leur lien, étaient considérés. Afin d'en évaluer la qualité rédactionnelle et philosophique, quatre domaines de compétences étaient examinés : linguistique, logique, créatif et réflexif. Ces domaines et les indicateurs correspondant ont été retenus sur la base de précédentes recherches ${ }^{34}$.

Les indicateurs pour apprécier la qualité linguistique des écrits étaient les suivants :

- le nombre de propositions constituant respectivement le texte et l'avant-texte (indicateurs quantitatifs),

- la présence d'une introduction (apparaissant à l'aide de formules telles que, par exemple, «Tout d'abord,...» ou «En premier, il y $a$... ») et celle d'une conclusion (visibles avec, par exemple, "Pour conclure ce texte, je peux dire $q u e . . . »$ ou encore «Merci de m'avoir suivi. ») (indicateurs qualitatifs).

La qualité logique était évaluée selon ces indicateurs :

\footnotetext{
${ }^{33}$ Auriac, E., \& Favart, M. (2007).

${ }^{34}$ Auriac-Slusarczyk, E., Thebault, C., Slusarczyk, B., Daniel, M. F., \& Pironom, J. (2018).

Auriac-Slusarczyk, E., Maire, H., Thebault, C., \& Slusarczyk, B. (2019).
} 
- le nombre de concepts (à la fois dans le texte et l'avant-texte ; par exemple : « imagination », « réalité » ou « émotions »),

- le nombre de postulats (par exemple : «Chaque rêve est unique, mais aucun n'est inutile »)

- le nombre d'hypothèses (avec des formulations telles que par exemple : «C'est comme si... » ou «Peut-être que...»),

- le nombre d'exemples impersonnels (c'est-à-dire, à caractère généralisant, universel, comme par exemple : "Les enfants rêvent d'avoir un amoureux riche, ou de quitter l'école, ou d'avoir un animal, alors que nos parents eux rêvent d'avoir un métier bien payé, une grosse voiture... en fait ils ne pensent pas comme nous »),

- la qualité des raisonnements (par exemple: "rêver est essentiel à la vie, d'ailleurs à tout âge on rêve, les plus petits rêvent déjà et les personnes âgées rêvent toujours»), et par

- la qualité des distinctions opérées entre concepts (par exemple : «Avec le temps, les attentes s'envolent, mais les rêves restent »).

La qualité créative des écrits était saisie à travers :

- la qualité de la divergence ${ }^{35}$ des idées émises (par exemple : «Dormir, c'est pour se reposer, mais je pense surtout une excuse pour laisser notre cerveau se faire envahir par les rêves et ainsi donner des réponses à nos questions » ou encore "Rêver est une chose légale »), et

- le nombre de métaphores (ou analogies) ${ }^{36}$ produites (par exemple : «Le rêve, c'est lorsque notre esprit s'envole» ou encore «...quel que soit notre rêve, il faut le prendre comme un message, une énigme à résoudre pour avancer»).

Enfin, la qualité réflexive révélait la distance de l'élève à son texte, et s'évaluait à l'aune d'indicateurs (reflétant pour les deux premiers l'attention portée à soi vs. la décentration) :

\footnotetext{
${ }^{35}$ En psychologie, il est d'usage de parler de « pensée divergente » pour qualifier la pensée à l'œuvre dans les situations où il faut trouver le maximum de solutions différentes à un même problème. Elle est d'ordinaire évaluée par des épreuves standardisées qui mesurent la capacité de l'individu à produire, dans un temps limité, de nombreuses idées à partir d'un point de départ simple (situation hypothétique, stimulus graphique, objet...). Les réponses fournies sont alors codées selon trois dimensions : la fluidité, qui renvoie à la quantité d'idées produites, la flexibilité, qui désigne le nombre de catégories dans lesquelles des idées peuvent être classées, et l'originalité, qui réfère à la rareté statistique relative de chaque idée proposée par rapport à une population de référence.

${ }^{36}$ Concernant les analogies, «Kant y voyait le ressort de la créativité et, pour ce qui est de Nietzsche, on se souvient de sa célèbre définition de la vérité comme d'une « armée mobile de métaphores » . (Hofstadter, D., \& Sander, E., 2013, p.30).
} 
- le nombre d'exemples personnels (c'est-à-dire des anecdotes de vie, comme par exemple : "Moi, il y a pas longtemps, j’ai regardé Roméo et Juliette, et après je rêvais de connaître un amour aussi fort qu'eux deux, mais sans mourir à la fin »),

- le nombre de pronoms personnels de la première personne du singulier (tels que «je», «moi », « mienne »...),

- le nombrede modalisateurs (les expressions du doute : «je ne sais pas », "peutêtre » ... ou de la nuance: «en quelque sorte», «dans certains cas », «quasi »...),

- le nombre de questionnements (par exemple : "Mettre sa vie en danger pour accomplir ses rêves est-il toujours bon?»),

- le niveau d'engagement personnel dans son texte, reflétés par le nombre de pronoms personnels de la première personne du singulier de prise en charge $d u$ discours (tels que «je pense que...» ou «à mon avis...») et le nombre de marqueurs évaluatifs (comme: "j'adore», «dommage» ou «heureusement»).

Pour chaque indicateur, les nombres bruts étaient divisés par le nombre total de propositions du texte (reflétant sa longueur), afin d'obtenir des ratios. Les résultats statistiques présentés ci-après se référeront à ces ratios. Le lecteur/la lectrice se reportera à des travaux antérieurs ${ }^{37}$ pour le détail de l'ensemble des variables testées. En annexes, deux exemples de copie dévoilent quelques-unes des marques scripturales considérées par l'étude menée.

Parallèlement à l'étude de l'impact du dispositif Philo \& Carto, il s'agissait aussi de repérer si le niveau scolaire en français moyen sur l'année et le genre des élèves pouvaient ou non moduler la qualité des écrits produits (les filles rédigeant souvent, au-delà du stéréotype, des écrits de meilleure facture que les garçons ${ }^{38}$ ).

\section{Les écrits philosophiques recueillis : un regard psychologique sur nos premiers résultats}

Afin d'étudier les processus cognitifs sous-jacents à l'écriture dans les textes d'élèves recueillis, trois effets majeurs ont été testés :

\footnotetext{
${ }^{37}$ Auriac-Slusarczyk, E., Thebault, C., Slusarczyk, B., Daniel, M. F., \& Pironom, J. (2018). Maire, H., \& Auriac-Slusarczyk, E. (à paraître).

${ }^{38}$ Auriac-Slusarczyk, E., Thebault, C., Slusarczyk, B., Daniel, M. F., \& Pironom, J. (2018).
} 
- l'effet du dispositif expérimental auquel étaient soumis les élèves (et variant selon les groupes : philosophant, semi-philosophant, contrôle),

- l'effet de leur niveau scolaire, en considérant, suite à des travaux précédents ${ }^{39}$, la moyenne en français comme variable d'appui,

- l'effet d'interaction entre ces deux facteurs.

Ce dernier effet correspond à la possibilité que l'effet d'un des facteurs varie de manière systématique selon les modalités de l'autre facteur ; autrement dit, dans notre cas, il s'agissait de voir si l'effet potentiel du dispositif pédagogique différait ou non de manière systématique selon le niveau en français des élèves. En complément à ce triptyque, l'effet du genre était simplement contrôlé. Sur le plan statistique, l'analyse des données à l'aide de modèles mixtes ${ }^{40}$ a été réalisée pour comparer les performances de nos trois groupes dans les quatre domaines ciblés et reflétés par les indicateurs de qualité rédactionnelle présentés ci-dessus. Nous exploiterons ici quelques résultats intéressants et prometteurs.

\section{3. a. Qu'observe-t-on ? Nos résultats}

L'étude des copies recueillies révèle qu'un transfert à l'écrit, ou du moins un écho de compétences similaires à celles développées à l'oral, s'opère. En effet, grâce aux analyses réalisées, l'avantage du groupe philosophant par rapport aux groupes semi-philosophant et contrôle est apparu dans certains domaines que nous avions ciblés (linguistique, logique, créatif et réflexif) : les élèves ayant bénéficié du dispositif Philo \& Carto ont produit des textes plus concis $\left(M_{\text {propositions }}=17.26\right.$, vs. $M_{\text {propositions }}=25.13$ et $M_{\text {propositions }}=20.21$ pour les groupes semiphilosophant et contrôle respectivement), comportant davantage de concepts $\left(M_{\text {concepts }}=1.54\right.$, vs. $M_{\text {conceps }}=1.10$ et $M_{\text {concepts }}=1.23$ pour les groupes semi-philosophant et contrôle respectivement $)$ et de postulats $\left(M_{\text {postulats }}=0.39\right.$, vs. $M_{\text {postulats }}=0.31$ et $M_{\text {postulats }}=0.34$ pour les groupes semi-philosophant et contrôle respectivement), étayés par plus d'images métaphoriques $\left(M_{\text {métaphores }}=0.06\right.$, vs. $M_{\text {métaphores }}=0.02$ et $M_{\text {métaphores }}=0.04$ pour les classes semi-philosophant et contrôle respectivement) mais paradoxalement moins d'expressions divergentes $\left(M_{\text {divergence }}=0.64\right.$, vs. $M_{\text {divergence }}=0.96$ et $M_{\text {divergence }}=1.17$ pour les groupes semiphilosophant et contrôle respectivement), et comportant beaucoup moins d'exemples personnels tels que des anecdotes de leur propre vie, suggérant une capacité amplifiée à la

\footnotetext{
${ }^{39}$ Auriac, E., \& Favart, M. (2007).

${ }^{40}$ Les analyses en «modèles mixtes » correspondent en psychologie à un type de traitement statistique dont la particularité est de prendre en compte à la fois les effets dits « fixes », c'est-à-dire des variables testées (ici, le dispositif expérimental et le niveau en français des élèves) et les effets dits « aléatoires » qui sont imputables aux participants comme sources de variabilité (ici, les élèves).
} 
décentration $\left(M_{\text {exemples personnels }}=0.03\right.$, vs. $M_{\text {exemples personnels }}=0.27$ et $M_{\text {exemples personnels }}=0.24$ pour les groupes semi-philosophant et contrôle respectivement). En revanche, ni l'introduction du dispositif Philo \& Carto dans les classes philosophant, ni la familiarisation avec la praxis philosophique en $6^{\text {ème }}$ et $5^{\text {ème }}$ dans les classes semi-philosophant, ne favorisait pas les compétences à introduire (pour les groupes philosophant, $M_{\text {introduction }}=46 \%$, vs. respectivement $M_{\text {introduction }}=28 \%$ et $M_{\text {introduction }}=38 \%$ pour les groupes semi-philosophant et contrôle) ou conclure un texte(pour les classes philosophant, $M_{\text {conclusion }}=30 \%$, vs. $M_{\text {conclusion }}=46 \%$ et $M_{\text {conclusion }}=31 \%$ pour les classes semi-philosophant et contrôle respectivement) .

Par ailleurs et sans surprise, nous avons constaté que le niveau moyen en français était corrélé positivement à certains des indicateurs retenus, notamment les indicateurs linguistiques (nombre de propositions, présence d'une introduction, présence d'une conclusion, $p s<.01$ ). En revanche, sur la quasi-totalité des autres indicateurs (logiques, créatifs, réflexifs), l'effet du dispositif et l'effet de la moyenne en français sur la qualité des écrits n'interagissaient pas ( $p$ s > .05); autrement dit, les bienfaits du dispositif étaient indépendants du niveau en français des élèves. Ce résultat est en harmonie avec l'idéal de démocratisation de l'art de raisonner souhaité par Lipman et ses continuateurs ${ }^{41}$. En outre, il suggère que la pratique orale de la DVP pourrait faciliter l'entrée dans l'écrit de certains élèves d'ordinaire plus inhibés sur ce terrain parfois perçu comme intimidant, car survalorisé culturellement dans le système scolaire français (" maîtriser la lettre, lettre du savoir, lettre du pouvoir, c'est la promesse d'une vie future respectable, non misérable $»^{42}$ ).

Notons toutefois une exception à ce résultat, qui porte sur l'emploi des métaphores : ici, les élèves philosophant utilisaient davantage de métaphores que les non philosophant ( $F$ (2, $143)=6.67, p<.002$ ), et ce d'autant plus que leur niveau en français était faible (pour l'interaction, $F(2,143)=12.02, p<.0001)$. On peut expliquer ce constat par un usage des métaphores comme « béquille » linguistique pouvant se substituer à un vocabulaire plus précis quand celui-ci vient à manquer, particulièrement chez les élèves avec un faible niveau en français. Ainsi, l'emploi des analogies dans une visée explicative (pour se faire comprendre des autres, et pas uniquement pour «décorer» son discours) par ces élèves s'accorde avec les travaux considérant le raisonnement analogique comme un véritable carburant de la pensée. En effet, l'analogie agit à la fois sur un mode économique, puisqu'elle permet de ramener l'inconnu au rang du connu et ainsi «de bénéficier, à faible coût cognitif, des acquisitions passées » ${ }^{43}$ et

\footnotetext{
${ }^{41}$ Maire, H., \& Auriac-Slusarczyk, E. (à paraître).

${ }^{42}$ Langlois, R. (2012).

${ }^{43}$ Hofstadter, D., \& Sander, E., (2013, p.36).
} 
vital, car «tout le monde dépend, sans pourtant s'en rendre compte, d'une dense avalanche de petites analogies quotidiennes $»^{44}$.

Enfin, contrairement à une précédente recherche ${ }^{22}$, les différences dans la qualité rédactionnelle des productions entre filles et garçons restaient ici marginales puisqu'elles concernaient uniquement la taille du texte (pour les filles, $M_{\text {propositions }}=25$; pour les garçons, $M_{\text {propositions }}=17$ ) et la présence de paragraphe ou de formules formant une introduction (pour les filles, $M_{\text {introduction }}=41 \%$; pour les garçons, $M_{\text {introduction }}=26 \%$ ), la fréquence de la présence d'une introduction restant à cet âge assez faible (entre $28 \%$ et $46 \%$ des élèves de notre échantillon).

\section{3. b. Quels effets propres au dispositif, quels effets globaux ?}

À la lumière de ces premiers résultats globaux encourageants, un bilan s'impose, notamment sur leur caractère généralisable ou non. Autrement dit, parmi les effets obtenus, lesquels relèveraient d'un noyau dur inhérent à toutes les variantes des dispositifs de praxis philosophique sous forme de discussions en communauté de recherche quelles qu'elles soient (ou presque), et lesquels seraient propres au dispositif Philo \& Carto spécifiquement étudié ici ?

La comparaison des performances des classes semi-philosophant avec celles des classes contrôle a permis de mettre en évidence les effets bénéfiques du protocole de praxis lipmanienne classique, et surtout leur résistance au temps (pour rappel, les élèves des classes semi-philosophant n'avaient pas pratiqué la discussion philosophique en communauté depuis plus de neuf mois lors des mesures écrites). En comparant uniquement ces deux groupes entre eux, on constate que ces élèves semi-philosophant fournissaient des écrits avec des raisonnements de meilleure qualité $\left(M_{\text {raisonnements }}=1.55\right)$ et plus d'exemples impersonnels, à portée universelle ( $\left.M_{\text {exemples impersonnels }}=0.52\right)$, que les élèves des classes contrôles $\left(M_{\text {raisonnements }}\right.$ $\left.=1.31 ; M_{\text {exemples impersonnels }}=0.45\right)$, n'ayant jamais bénéficié de praxis philosophique.

Conjointement, la comparaison des performances des classes philosophant (praxis du dispositif Philo \& Carto, sans aucune sensibilisation antérieure) avec celles des classes semiphilosophant a mis en lumière des effets propres au dispositif Philo \& Carto. Il apparaît que les élèves philosophant avec ce dispositif écrivaient des textes contenant davantage de concepts, de postulats et de métaphores (pour rappel, $M_{\text {concepts }}=1.54 ; M_{\text {postulats }}=0.39 ; M_{\text {métaphores }}=0.06$ ) que les élèves semi-philosophant (pour rappel, $M_{\text {concepts }}=1.10 ; M_{\text {postulats }}=0.31 ; M_{\text {métaphores }}=$

\footnotetext{
${ }^{44}$ Hofstadter, D., \& Sander, E., (2013, p.34).
} 
0.02). Parce que le dispositif Philo \& Carto invite explicitement les élèves à associer de façon systématique des concepts abstraits (la différence, l'amour, l'attachement, la prudence, le destin, etc. ${ }^{28}$ ) à des éléments sensibles et concrets (le ciel, le sol, le nuage, le mur, etc.), on peut penser qu'il favorise particulièrement le recours aux concepts et la mobilisation du procédé métaphorique. Le lecteur trouvera des détails dans un article précédent ${ }^{45}$ mettant en valeur cette créativité métaphorique à l'œuvre grâce au dispositif Philo \& Carto.

\section{Variantes à partir du dispositif Philo \& Carto}

Soutenus par ces résultats prometteurs, nous invitons les enseignantes et enseignants désirant expérimenter la pratique de la $\mathrm{P} 4 \mathrm{C}$ dans leurs classes à s'essayer à plusieurs variantes de dispositifs, voire à ne pas hésiter à faire preuve d'improvisation et de créativité. Si le protocole lipmanien classique offre la sécurité d'un dispositif solide, expérimenté et positivement évalué dans des contextes variés et auprès de publics divers, si notre protocole mêlant art et philosophie peut également être investi, d'autres variantes existent et des innovations maitrisées sont toujours, en la matière, bienvenues.

Le récent ouvrage publié par François Galichet, visant le philosopher à tout âge, en appelant au sensible, au subjectif, à l'interprétation, nous paraît faire écho au principe fondateur du protocole Philo \& Carto. C'est l'approche interprétative qui est au cœur des principes de Galichet et du dispositif Philo \& Carto. Ainsi, la visée d'un dialogue philosophique n'est pas de «démontrer un énoncé (...) [mais de] scruter le vécu ${ }^{46}$. En effet, les vertus intellectualisantes parfois attribuées systématiquement à la pratique philosophique (via les habiletés cognitives à conceptualiser, problématiser, argumenter) ne lui étant pas exclusives, il convient d'intégrer au maximum la composante expérientielle et singulière, voire intime, qui se révèle également dans l'écrit philosophique tel que nous l'avons décrypté. Cet ancrage subjectif singulier, à l'œuvre dans les écrits comme dans les dialogues philosophiques oraux, active un élan vers le commun, l'universel, l'intérêt étant porté «non pas [au] vécu individuel et singulier de chacun, mais [au] vécu générique de l'homme en tant que sujet universalisant d'une expérience $»^{47}$. Aborder la praxis philosophique dans cette optique assure une grande attention portée à la mobilisation d'implicites, d'espaces latents, perçus mais non encore verbalisés et qui peuvent ensuite cheminer vers une mise en mots.

\footnotetext{
${ }^{45}$ Auriac-Slusarczyk, E, Thebault. C, \& Slusarczyk, B. (2018).

${ }^{46}$ Galichet, F. (2019).

${ }^{47}$ Galichet, F. (2019).
} 
Une telle approche interprétative ${ }^{48}$ fait écho aux travaux de psychologie qui revisitent, dans des champs conceptuels et selon des objectifs d'étude parfois éloignés, l'épineuse question des liens entre émotion et cognition. Nous citerons deux grandes familles de ces travaux, complémentaires sans être antagonistes, qui peuvent inspirer les enseignantes et enseignants. Certaines recherches, issues de la neuropsychologie, arguent que l'émotion façonne la pensée et s'attachent à « réhabiliter » l'émotion comme partie intégrante des processus de raisonnement et sous-bassement commun avec la conscience ${ }^{49}$. Lipman présupposait déjà que «les émotions forment et dirigent les pensées, les charpentent, leur confèrent un équilibre, un point de vue ou mieux, plusieurs. Sans émotion, la pensée serait plate et dénuée d'intérêt ${ }^{50}$. D'autres recherches inversent le mouvement et montrent que la pensée façonne l'émotion. Certaines habiletés cognitives, telles que la pensée critique, modifiant la compréhension ou l'interprétation d'une situation, moduleraient alors le vécu émotionnel ${ }^{51}$.

Les émotions, et ainsi tous les supports pédagogiques permettant leur émergence, constituent un ingrédient nécessaire pour raisonner. Même lorsqu'elles «débordent » l'individu, et sont sources de nombreux biais de jugement ou erreurs d'évaluation, elles permettent à l'individu de naviguer psychiquement. C'est pourquoi la pensée critique, telle que pratiquée dans les DVP dans la lignée lipmanienne «fournit un espace pour « sélectionner » les émotions et les sentiments et ainsi reconnaître ceux qui sont les plus appropriés pour une situation donnée $»^{42}$.

Nous l'évoquions plus haut, les enseignantes et enseignants doivent, selon notre approche, rester inventifs en matière de praxis philosophiques. Si le protocole testé Philo \& Carto ambitionne une forme particulière de mise en correspondance de l'Art et du Philosopher, maintes pistes peuvent être explorées. Ainsi les supports musicaux peuvent donner lieu à investissement. Des classes de collège, une fois rodés aux principes lipmaniens de respect de la pensée et d'écoute d'autrui, de curiosité et de mobilisation de leur faculté de questionnement, et ce de manière permanente, sauront se saisir de toutes les opportunités pédagogiques. Notre conclusion en développera quelques-unes.

\section{Conclusion, enjeux et pistes pour les éducateurs}

\footnotetext{
${ }^{48}$ Galichet, F. (2019).

${ }^{49}$ Damasio, A.R. (1999).

${ }^{50}$ Lipman, M. (2006, p.250).

${ }^{51}$ Lafortune, L., \& Robertson, A. (2005, p.64).
} 
Au plan formel, il est à souligner que les mesures effectuées dans cette étude, restent des photographies de compétences écrites prélevées à un instant unique ; elles mériteraient, pour le psychologue, d'être répétées. On pourrait alors, en reproduisant puis en variant les conditions, dessiner des trajectoires différentielles et tester le caractère plus ou moins durable des bienfaits de la praxis philosophique Philo \& Carto. Parmi les variantes, nous suggérons aux enseignantes et enseignants de varier les supports culturels à disposition, des œuvres très (trop ?) circonscrites par le patrimoine culturel, avec des auvres plus inédites (art contemporain), voire plus polémiques (journaux, clichés prélevés sur Internet, illustrations moins ancrées dans la culture et peut-être moins intimidantes pour les élèves), et ce pour deux raisons. D'une part, on doit garder à l'esprit que les élèves n'appartiennent pas tous, ou de la même manière, à une filiation culturelle dominante telle qu'elle est pourtant souvent choisie par les enseignantes et enseignants (repli sur des œuvres d'art connues du patrimoine). Il peut alors être judicieux d'investir le champ d'œuvres moins courues, telles les peintures du courant des vanités ${ }^{52}$, qui offre l'avantage de convier les élèves, comme leurs professeurs, à sortir des sentiers battus. D'autre part, c'est aussi la variation des supports déclencheurs de discussion qui amène les élèves à explorer et à cheminer cognitivement de manière plus risquée, se séparant ainsi d'habitus qui les ont parfois conduit à resserrer leurs compétences sur un noyau utile, mais peu créatif.

Conjointement, à la manière du «croyant critique » Freddy Mortier ${ }^{53}$, nous pensons qu'une diffusion de la philosophie pour enfants, pour qu'elle soit féconde et pérenne, doit continuellement pouvoir répondre à la question de l'efficacité : est-ce que ça marche ? Certes, le dispositif miracle, aux effets à la fois de grande ampleur, multiples, transférables et durables, n'existe pas. Certes, évaluer l'efficacité d'un dispositif pédagogique est toujours délicat, tant celui qui le met en œuvre peut adopter une attitude moins critique et plus complaisante, qui peut distordre l'évaluation et artificiellement grossir l'ampleur de (pré)supposés bienfaits. C'est pourquoi, l'essaimage des pratiques de praxis philosophique par la discussion gagne à s'accompagner de travaux de recherche visant à saisir et délimiter quelques effets. Ce fut le cas de notre entreprise collaborative entre chercheurs et enseignants de collège. Dans cette optique, la psychologie du développement, la psychologie cognitive et la psychologie différentielle peuvent être mobilisées comme d'utiles ressources, à la fois dans leurs concepts, leurs méthodes et leurs outils, mais aussi, nous espérons l'avoir esquissé ici, pour favoriser la créativité des

\footnotetext{
52 Voir par exemple la démarche de Peyronnet, A. (2001).

${ }^{53}$ Mortier, F. (2005).
} 
enseignantes et enseignants qui seront les porteurs du renouveau dans les classes du XXI ${ }^{\text {ème }}$ siècle déjà bien entamé.

Pour conclure, il peut être utile de rappeler que les dispositifs de praxis de la philosophie par la discussion brillent (aussi) par leur simplicité. En effet, ils ne nécessitent ni matériel commercialisé, particulier ou coûteux. Ils évitent ainsi l'asservissement décourageant à la technique et/ou à l'argent. Ni diplôme en philosophie ou ni connaissances disciplinaires préalables ne sont obligatoires; de telles connaissances pourraient même constituer des entraves à la conduite d'oraux riches, libres et inventifs. Les élèves, les enfants, les adolescents nous surprennent et doivent conserver cette part de renouvellement des esprits, en favorisant l'émergence de pensées adaptées à leur monde ${ }^{17}$.

La formation à la maîtrise des protocoles cadrant les expériences de praxis philosophique à l'école, se réduit actuellement à quelques demi-journées. À l'heure de l'envahissement numérique, il est à propos de rappeler la possibilité de se libérer d'une telle omniprésence entravant parfois les élans créatifs, afin que des enseignantes et enseignants, peutêtre encore hésitants, se lancent dans des pratiques philosophiques avec leurs classes, en réinvestissant le bien le plus précieux de l'être humain : sa capacité de pensée. 


\section{Références bibliographiques}

Auriac, E., \& Favart, M. (2007). Passage d'un avant-texte au texte dans des écrits scolaires de type argumentatif. Langue Française, 115(3). 69-83.

Auriac-Slusarczyk, E., \& Colletta J.-M. (2013). Les ateliers de philosophie : une pensée collective en acte. Clermont Ferrand : Presses Universitaires Blaise Pascal.

Auriac-Slusarczyk, E., \& Maire, H. (à paraître). Sur les pas de Lipman, philosopher à l'école. Une histoire scientifique à connaître. In J.-P. Simon, A. Fournel, S. Lagrange-Lanaspre, J.-M. Colletta, \& E. AuriacSlusarczyk (Eds.), Philosopher avec les enfants, fabrique de l'apprendre, fabrique du savoir. Clermont Ferrand : Presses Universitaires Blaise Pascal.

Auriac-Slusarczyk, E., Maire, H., Thebault, C., \& Slusarczyk, B. (2019). Improving the quality of philosophical writing: Experimentation on the first philosophical compositions of 12 and 13-year old pupils. Educational Review, 1-23. doi:10.1080/00131911.2018.1546166

Auriac-Slusarczyk, E, Thebault. C, \& Slusarczyk, B. (2018). Mind mapping and philosophy in junior high school. A comparative study of initial philosophical writing in classes of 8 th grade pupils used and unused to philosophically directed discussion. Creative Education, 9, 1312-1331, doi:10.4236/ce.2018.99098

Auriac-Slusarczyk, E., Thebault, C., Slusarczyk, B., Daniel, M. F., \& Pironom, J. (2018). Premiers écrits philosophiques. Productivité conceptuelle et créativité rédactionnelle du CE2 au CM2. Bulletin de Psychologie, 3, 671-690.

Beausoleil, J., \& Daniel, M.-F. (1991). L’identification des dimensions philosophiques dans les dialogues des élèves, Arrimages, 7/8, 17-24.

Choulet, P., Folscheid, D., \& Wunenbuger, J.-J. (1992). Méthodologie philosophique. Paris : Presses Universitaires de France.

Colom, R., Moriyón, F. G., Magro, C., \& Morilla, E. (2014). The long-term impact of Philosophy for Children: A longitudinal study (preliminary results). Analytic Teaching and Philosophical Praxis, 35(1), 50-56.

Damasio, A.R. (1999). Le sentiment même de soi. Corps, émotions, conscience. Paris : Odile Jacob.

Daniel, M.-F. (2003). Une coopération de haut niveau : l'exemple de la philosophie pour enfants. In E. AuriacPeyronnet (Ed.). Je parle, tu parles, nous apprenons (pp. 239-250). Bruxelles : DeBoeck Université.

Daniel, M.-F. (2005). Présupposés philosophiques et pédagogiques de Matthew Lipman et leurs applications. In C. Leleux, La philosophie pour enfants : Le modèle de Matthew Lipman en discussion (pp.34-45). Bruxelles : De Boeck.

Dewey, J. (1958). Experience and nature. New York: Dover Publications.

Dewey, J. (1963). Experience and education. New York: Macmillan Publishing. Fenoglio, I., \& BoucheronPétillon, S. (2002). Processus d'écriture et marques linguistiques : nouvelles recherches en génétique du texte. Paris : Larousse.

Galichet, F. (2007). La philosophie à l'école. Paris : Milan.

Galichet, F. (2019). Philosopher à tout âge. Paris : Vrin.

García-Moriyón, F., Rebollo, I., \& Colom, R. (2005). Evaluating philosophy for children: A meta-analysis. Thinking: The Journal of Philosophy for Children, 17(4), 14-22.

Giménez-Dasí, M., Quintanilla, L., \& Daniel, M.-F. (2013). Improving emotion comprehension and social skills in early childhood through philosophy for children. Childhood and Philosophy, 9(17), 63-89.

Gregory, M.-R., Haynes, J., \& Murris, K. (2016). Philosophy for Children: an educational and philosophical movement. In M.R. Gregory, J. Haynes, \& K. Murris, The Routledge International Handbook of Philosophy for Children (pp. xxi-xxxi). New York: Routledge. 
Heron, G., \& Cassidy, C. (2018). Using practical philosophy to enhance the self-regulation of children in secure accommodation. Emotional and Behavioural Difficulties, 23(3), 254-269.

Hofstadter, D., \& Sander, E. (2013). Surfaces and essences: Analogy as the fuel and fire of thinking. Basic Books.

Lafortune, L., \& Robertson, A. (2005). Une réflexion portant sur les liens entre émotions et pensée critique. In L. Lafortune, M.-F. Daniel, P.-A. Doudin, F. Pons \& O. Albanese (Eds.), Pédagogie et psychologie des émotions. Vers la compétence émotionnelle (pp. 61-84). Québec : Presse de l'Université du Québec.

Langlois, R. (2012). Les précurseurs de l'oralité scolaires en Europe : De l'oral à la parole vivante. Rennes : Presses Universitaires de Rennes.

Lévine, J. (2007). La notion de «monde philosophique des enfants » : utopie ou nécessité ? Présupposés, place, limites ? In M. Tozzi (Ed.), Apprendre à philosopher par la discussion. Pourquoi ? Comment ? (pp. 95108). Bruxelles : De Boeck Université.

Lévine, J., Chambart, G., \& Sillam, M. (2005). Essai sur le monde philosophique de l'enfant. Le dialogue Moi Monde-Instance Monde. In C. Leleux, La philosophie pour enfants : Le modèle de Matthew Lipman en discussion (pp.71-93). Bruxelles : De Boeck.

Lipman, M. (1991). Thinking in education. Cambridge: Cambridge University Press.

Lipman, M. (2006). A l'école de la pensée. Enseigner une pensée holisitique. Bruxelles : De Boeck et Larcier.

Lipman, M., Sharp, A. M., \& Oscanyan, F. S. (1980). Philosophy in the classroom. Philadelphia, PA: Temple University Press.

Maire, H., \& Auriac-Slusarczyk, E. (à paraître). Premiers écrits philosophiques en $4{ }^{\text {ème }}$. In J.-P. Simon, A. Fournel, S. Lagrange-Lanaspre, J.-M. Colletta, \& E. Auriac-Slusarczyk (Eds.), Philosopher avec les enfants, fabrique de l'apprendre, fabrique du savoir. Clermont Ferrand : Presses Universitaires Blaise Pascal.

Millett, S., \& Tapper, A. (2012). Benefits of collaborative philosophical inquiry in schools. Educational Philosophy and Theory, 44(5), 546-567. doi:10.1111/j.1469-5812.2010.00727.x

Mortier, F. (2005). Etude d'évaluation : la méthode de Matthew Lipman comme moyen de développement. In C. Leleux, La philosophie pour enfants : Le modèle de Matthew Lipman en discussion (pp.47-69). Bruxelles : De Boeck.

Peyronnet, A. (2001). Sensibiliser au tragique de l'existence. Diotime, 10. En ligne: http://www.educrevues.fr/DIOTIME/AffichageDocument.aspx?iddoc=38644.

Rispail, M., Calistri, C., Martel, C., \& Bomel-Rainelli, B. (2007). Apprendre à parler, apprendre à penser. Les ateliers de philosophie. Nice : Sceren Edition.

Sasseville, M. (2005). Des enfants philosophent [DVD 1 et 2]. Laval, Québec : Université Laval.

Schneuwly, B. (1994). Vygotsky, Freinet et l'écrit. In P. Clanché, E. Debarbieux \& J. Testanière, La pédagogie Freinet. Mises à jour et perspectives (pp. 313-324). Talence : Presses Universitaires de Bordeaux.

Slusarczyk, B. et collaborateurs (2017). Philosoph' arts. Cartographie philosophique à l'usage des collégiens. Clermont Ferrand : Un, deux... quatre.

Thebault, C. (2015). Une expérience d'atelier Philo \& Carto. Diotime, 65, 4-7.

Thebault, C. (à paraître). Le dispositif Philosophie et Cartographie. In J.-P. Simon, A. Fournel, S. LagrangeLanaspre, J.-M. Colletta, \& E. Auriac-Slusarczyk (Eds.), Philosopher avec les enfants, fabrique de l'apprendre, fabrique du savoir. Clermont Ferrand : Presses Universitaires Blaise Pascal.

Tozzi, M. (2005). Lipman, Lévine, Tozzi: différences et complémentarités. In C. Leleux, La philosophie pour enfants : Le modèle de Matthew Lipman en discussion (pp.95-115). Bruxelles : De Boeck.

Trickey, S., \& Topping, K. J. (2006). Collaborative philosophical enquiry for school children: Socio-emotional effects at 10-12 years. School Psychology International, 27(5), 599-614. 


\section{Annexes :}

\section{Exemples de copies d'élèves du groupe philosophant}

Copie A (fille, 13 ans) :

Ee rêre peut être plusievers chooes, peut-ettre tout d'alarerd un présage de láven ou un douloureux passé qui rous hande l'esprit. Pour moi les rêves Cou les cauchemars même si ilo font peur) nous appor bent forcément quelques chases de bien de notre vie. Je pense que quelque soit notre rôve, ilfaut le prendre comme un message, une enigmeà réscu dre paur avancer dans le droit chermin. Hème les persannes les plus charceux ont forcément des uostacle dans Bewr vie au alors, meime las personnes les plus respectueux et aimant font du mal parfois aux gens, chacun de nous fait des evreurs, c'est hu main, mul n'est parfait! 'Et a mon sens c'est à cela que servent bes revers, a voir dedarns ce qu il fout corriger et améliorer pour réparer les erreurs de notre vie! Enaque rêve est unique mais au aum n'est inutule! E'est vrai sinous rêvons (a faisons des cauchemars) éest bien que c'est neces saire sinon on ne rêverait pas. Et mêma plus loin, dormir céstvrai c'est pouer se reposer mais je pense surtout une exause pour laissen notre corvacu se faire envahir par les rôve et ainsi donner raiponse à nos questions, ainsi que des solutions à nes problèmes. Eles rêres et les cauchemares bien-sêr sont de granroles enigmes sur leur utilité. An l'ignore souvent mais je pense que nous devons écouter nos réres, ibs sont comme la seul et unique dé Cissue) de notre vie avec ses problémes, Put-être même qu ils prévoient léavene et que peu de pensonne arrivent i la comprenobre à par las médiomes! Mềne si les réves font parfois mal, ils servont er nous aident dans toutes 
difficultés que nous pourons sencontror... Et puis nous disons sourent que seul la vérité fait mal et surtout quand elle ert arutale! Don r vilà pour moi les rêres ou les eau chermards sont des mersages auxquelles ils faut écouter les conseils. Ce vais parber mainbenant d une deuxieme sorte de zêre, celvi de lonvie, de l'objectif ou du but que nous pourons se fixer dans noter vie pour avancor. . Je pense qu'ilest impontant d'avoir un dojectif dans sa vie, cla nous rend plus fort et nous donne plus (t) confrance en soi: Parfais nos rêres sont tòs originals et ćest surtout dans ces cas la qu il est difficile de śy accroché ear tout le monde nous dit que c'est impossible, alors que beaucaup d'entre nous ont besoin de soution que de décour agement, mais malgrès l'avis des autres, c'est le notre qui compte car c'est notre objecty etpas celsi des autres 'ela seul chose que je peux vous dire est acurochez-vaus à vos buts sivousvoulag qu'ils se réalisent Ekaqun de nous est difpérents et a des envies différentes et il Paut respecter ceex des autres sivaus vou by que les autres respect les vôtres. Pour con lure ce texte je peux dire que lavie noues a donné ces rèves quel qu'ils sont et qu ils ne faut pas bos igmorer à cause des autres our parce qu' on a peur: Ayez confiance en vous et trout ira dans votresens... 
Copie B (garçon, 13 ans) :

DEBUT. Un rêve est une wision de la réalité parfois il se réalise parfois, it defois non. Cela nousdonne desidées pour notre avenir. "Rêre" est un mot bien vastes mais powr les petits rêver vent dire dormir. Now nous revons de personenesque lón connait ou de chosesque l'on a rue dans la journée et quand les images s'associe à des monstires ou deschoses qui hont peur, on fait descauchemars. Le reve est un moment or l immagination prend $l_{e}$ contrôle de notre corps. Rêver c'est voir peu être le futur, c'et pensé à l'avenir, à notreanenir. It sans rêve pas d'immagination. Le rềve cést lavie et la vie cés un rêve blanc que nous devons écrire. In jour \&̀ai rềvé que: J'etait passé dans uneautru dimension et je me suis retrowé lans deo ruines. J'etait entouré par des personnages ficitis, il y avait Merlin l'enchanteur, la fée lockette et la reine des neiges 